\title{
Observation of Double-Dome Superconductivity in Potassium-Doped FeSe Thin Films
}

\author{
Can-Li Song, ${ }^{1,2}$ Hui-Min Zhang, ${ }^{1}$ Yong Zhong, ${ }^{1}$ Xiao-Peng Hu, ${ }^{1}$ Shuai-Hua Ji, ${ }^{1,2}$ Lili Wang, ${ }^{1,2}$ \\ $\mathrm{Ke} \mathrm{He},{ }^{1,2}$ Xu-Cun Ma, ${ }^{1,2, *}$ and Qi-Kun Xue ${ }^{1,2, \dagger}$ \\ ${ }^{1}$ State Key Laboratory of Low-Dimensional Quantum Physics, Department of Physics, Tsinghua University, Beijing 100084, China \\ ${ }^{2}$ Collaborative Innovation Center of Quantum Matter, Beijing 100084, China
}

(Received 6 November 2015; published 11 April 2016)

\begin{abstract}
We report on the emergence of two disconnected superconducting domes in alkali-metal potassium- (K-) doped FeSe ultrathin films grown on graphitized $\mathrm{SiC}(0001)$. The superconductivity exhibits hypersensitivity to $\mathrm{K}$ dosage in the lower- $T_{\mathrm{c}}$ dome, whereas in the heavily electron-doped higher- $T_{\mathrm{c}}$ dome it becomes spatially homogeneous and robust against disorder, supportive of a conventional Cooper-pairing mechanism. Furthermore, the heavily K-doped multilayer FeSe films all reveal a large superconducting gap of $\sim 14 \mathrm{meV}$, irrespective of film thickness, verifying the higher- $T_{\mathrm{c}}$ superconductivity only in the topmost FeSe layer. The unusual finding of a double-dome superconducting phase is a step towards the mechanistic understanding of superconductivity in FeSe-derived superconductors.
\end{abstract}

DOI: 10.1103/PhysRevLett.116.157001

Interface-enhanced high temperature superconductivity in single-layer FeSe films on $\mathrm{SrTiO}_{3}\left(\mathrm{FeSe} / \mathrm{SrTiO}_{3}\right.$ films $)$ [1,2] was discovered in 2012 and exhibits an unexpected

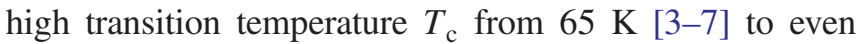
$109 \mathrm{~K}$ [8]. So far, this subject has experienced a tremendous burst of theoretical and experimental activities in the superconductivity community [2-18], because it offers an unprecedented opportunity in the quest of the mysterious mechanism behind Cooper pairing in high- $T_{\mathrm{c}}$ superconductors [1]. Using an angle resolved photoemission spectroscopy (ARPES) technique, it was immediately confirmed that the superconducting single-layer FeSe/ $\mathrm{SrTiO}_{3}$ films are sized of a rather simple Fermi surface topology with only electronlike band(s) around the zone corner $M[3-5,14]$. This has posed a massive challenge to the ever prevailing pairing paradigm of iron-based superconductors (Fe SCs), in which the repulsive interband interaction between the holelike bands around the zone center $\Gamma$ and the electron bands around $M$ leads to strong spin fluctuation and consequently a sign-reversing $s$-wave state $\left(s_{ \pm}\right.$pairing symmetry) [19-21]. Subsequent theoretical efforts to tentatively interpret this unwonted phenomenon have extended the $s_{+}$pairing model and generated an "incipient" $s_{ \pm}$-wave, a nodeless $d$-wave, and a more subtle sign-changed $s$-wave state between two $M$ near hybridized electron pockets [21], but have received little attention. Alternatively, the conventional Cooper pairing mechanism based on a phonon scenario from either FeSe itself [10,12] or across the interface $[14,16,18,22]$, in conjunction with electron transfer from $\mathrm{SrTiO}_{3}$ to $\mathrm{FeSe}$ films, has been proposed and attracts increasing attention $[1,14,22]$. This is further supported by the recent observation of plain $s$-wave superconductivity with no sign change, rather than a spin fluctuation driven $s_{ \pm}$-wave state and its extensions in $\mathrm{FeSe} / \mathrm{SrTiO}_{3}$ films [23].
On the other hand, the recent demonstrations of high temperature superconductivity in heavily electron-doped FeSe films or flakes through an alkali-metal potassium (K) [24-26] and liquid-gating technique [27,28] raise new concerns over the superconductivity in FeSe-related materials. Considering alkali metals and small molecules intercalated $\mathrm{FeSe}$ compounds with similarly high $T_{\mathrm{c}}$ $(\sim 40 \mathrm{~K})$ as well [29-32], one central issue that naturally arises is whether the heavily electron-doped FeSe compounds including single-layer $\mathrm{FeSe} / \mathrm{SrTiO}_{3}$ films represent novel superconductors with completely distinct pairing mechanism (e.g., phonon-mediated electron paring) from Fe SCs, or whether they are merely some derivatives of heavily electron-doped Fe SCs. In order to address this question, it is highly tempting to study systematically how the superconductivity is altered from a low- $T_{\mathrm{c}}$ phase in undoped parent $\mathrm{FeSe}$ to a high- $T_{\mathrm{c}}$ phase in heavily electron-doped FeSe with increasing electron doping level $x$. However, all previous attempts at establishing such a phase diagram were conducted either in nonsuperconducting strained multilayer $\mathrm{FeSe} / \mathrm{SrTiO}_{3}$ films [24-26], or in liquid-gating tuned $\mathrm{FeSe}$ thin flakes suffering from a significant inhomogeneity of the electron-density distribution [27], which have severely hampered the clear identification of the FeSe superconducting phase diagram.

Herein we report on such a phase diagram by exploring superconductivity in thickness-controlled FeSe ultrathin films grown on a graphitized $\mathrm{SiC}(0001)$ substrate [33-35] with increasing surface $\mathrm{K}$ dosage using scanning tunneling microscopy or spectroscopy (STM or STS). This allows for a direct probing of the superconducting order parameter at the nanoscale, thus avoiding the macroscopic integral measurements involved in ARPES and transport techniques $[24,25,27]$. Meanwhile, the nearly "free-standing" FeSe films on graphitized $\mathrm{SiC}(0001)$ [34] rule out the possible 

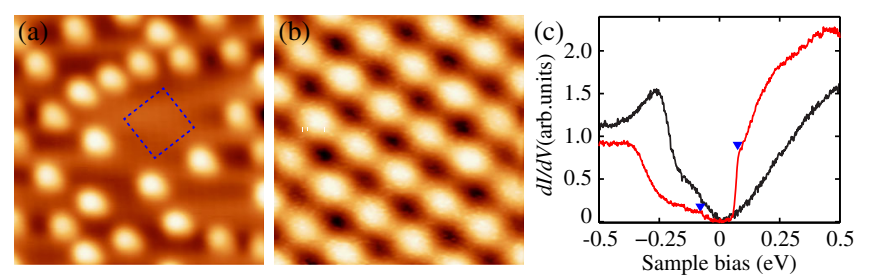

FIG. 1. (a) STM topographic image of K-doped FeSe/ $\mathrm{SiC}(0001)$ films $(10 \mathrm{~nm} \times 10 \mathrm{~nm}, \quad V=1.0 \mathrm{~V}, \quad I=50 \mathrm{pA})$. (b) Atomically resolved topography measured in K-free region $(2 \mathrm{~nm} \times 2 \mathrm{~nm}, V=2 \mathrm{mV}, I=100 \mathrm{pA})$, marked by the dashed square in (a). (c) Differential conductance $d I / d V$ spectra of FeSe films before (black curve) and after (red curve) a surface dose of $0.205 \mathrm{ML}$ K. The two triangles denote the edges of the accidental gap between the electron and hole pockets around the $\Gamma$ point. The tunneling gap is set at $V=0.5 \mathrm{~V}, I=100 \mathrm{pA}$. The lock-in bias modulation has a magnitude of $10 \mathrm{meV}$.

interruption of the lattice mismatch-caused epitaxial strain and zebralike stripes in multilayer $\mathrm{FeSe} / \mathrm{SrTiO}_{3}$ films $[5,6,36]$. Our experiments were carried out on a Unisoku ultrahigh vacuum cryogenic STM system equipped with molecular beam epitaxy (MBE) equipment for in situ FeSe film growth. High-quality superconducting FeSe/ $\mathrm{SiC}(0001)$ thin films with varying thicknesses were prepared following the well-established codeposition method, as described in our previous reports [33,34]. $\mathrm{K}$ atoms were then evaporated from a well-outgassed getter source (SAES group) onto FeSe films kept at $\sim 150 \mathrm{~K}$. Prior to the STM or STS measurements at $4.3 \mathrm{~K}$, polycrystalline PtIr tips were cleaned by electron-beam heating and then calibrated on MBE-grown $\mathrm{Ag} / \mathrm{Si}(111)$ films. Tunneling spectra were acquired using a standard lock-in technique with a small bias modulation of $0.2 \mathrm{mV}$ at $966 \mathrm{~Hz}$, unless otherwise specified.

Figure 1(a) depicts a constant-current topographic image of $\mathrm{K}$-doped $\mathrm{FeSe} / \mathrm{SiC}(0001)$ films with a nominal $\mathrm{K}$ dosage of about 0.038 monolayer (ML). Here $1 \mathrm{ML}$ is defined as the $\mathrm{Se}$ atomic number density at the topmost Se layer $\left(\sim 7 \times 10^{14} / \mathrm{cm}^{2}\right)$. Evidently, individual isolated $\mathrm{K}$ adatoms are randomly distributed at the surface. The absence of a $\mathrm{K}$ dimer, multimer, or cluster hints at a strong repulsive interaction among the ionized $\mathrm{K}$ adatoms because of electron transfer from $\mathrm{K}$ to $\mathrm{FeSe}$ [37], consistent with previous reports [24-26]. Enlarged STM images on any regions with sparse K adatoms [e.g., Fig. 1(b)] all reveal an untouched Se lattice, irrespective of how we postanneal the samples at the elevated temperature $\left(<400{ }^{\circ} \mathrm{C}\right)$. Plotted in Fig. 1(c) are the spatially averaged differential conductance $d I / d V$ spectra over a wide energy range $(-0.5-0.5 \mathrm{eV})$, acquired on FeSe before and after a surface dose of $\sim 0.205$ ML K, respectively. The $\mathrm{K}$ adsorption does not lead to a simple rigid shift in the band structure of the $\mathrm{FeSe} / \mathrm{SiC}(0001)$ films, but instead suppresses strongly the spectral weight near the Fermi level $E_{F}$. The resulting electronic structure resembles that of single-layer $\mathrm{FeSe} / \mathrm{SrTiO}_{3}$ films in a prominent manner, caused primarily by the accidental gapping between the electron and hole pockets around the $\Gamma$ point $[3-5,14,17]$. The gap size, defined as the energy separation between two gap edges [indicated by the blue triangles in Fig. 1(c)], is measured to be around $135 \mathrm{meV}$, quite close to the value of $140 \mathrm{meV}$ in single-layer $\mathrm{FeSe} / \mathrm{SrTiO}_{3}$ films [17]. All of these findings indicate that a systematic spectral survey of $\mathrm{K}$-doped $\mathrm{FeSe} / \mathrm{SiC}$ films will shed some critical insights into superconductivity in FeSe-derived superconductors.

Enumerated in Fig. 2 are a series of topographies and much smaller-energy-ranged $d I / d V$ spectra in multilayer $\mathrm{FeSe} / \mathrm{SiC}$ films as the $\mathrm{K}$ dosage is gradually increased. As anticipated, the parent FeSe film in Fig. 2(a) exhibits a single dominant superconducting gap $\Delta \sim 2.2 \mathrm{meV}$ [Fig. 2(f)], matching exactly with previous studies [33-35]. Given that $T_{c} \sim 9.3 \mathrm{~K}$ [34], this leads to a reduced gap $2 \Delta / k_{\mathrm{B}} T_{\mathrm{c}} \sim 5.5$, indicating strong-coupling superconductivity in the parent and undoped FeSe. As the dose is increased, individual isolated $\mathrm{K}$ adatoms tend to pile up together [Figs. 2(d) and 2(e)], analogous to $\mathrm{K}$-coated $\mathrm{FeSe} / \mathrm{SrTiO}_{3}$ films [26,38]. Quite interestingly, a considerable amount of $U$-shaped spectral weight depletion or loss, with two sets of $E_{F}$-symmetric peaks (black arrows) at the higher energy positions of $\sim 14 \mathrm{meV}$ and $8.5 \mathrm{meV}$, respectively, is invariably revealed in heavily K-doped FeSe/SiC films [Fig. 2(j)]. Such a double-gap superconductivity, which originates from a multiband character [39], bears a striking resemblance to those observed in heavily electron-doped FeSe-derived superconductors [1,24-32], signalling the occurrence of high- $T_{\mathrm{c}}$ superconductivity in heavily K-doped multilayer $\mathrm{FeSe} / \mathrm{SiC}$ films. This is supported by the recent temperature-dependent ARPES measurements of a K-coated FeSe single crystal: the superconducting gap vanishes near $T_{\mathrm{c}} \sim 25 \mathrm{~K}$ [40].

Furthermore, we found that with increasing $\mathrm{K}$ dosage, the stoichiometric parent $\mathrm{FeSe}$ with a low $T_{\mathrm{c}}$ (or equivalently small $\Delta$ ) does not evolve monotonically into the high- $T_{\mathrm{c}}$ phase; rather, its superconductivity weakens first [Fig. 2(g)], vanishes entirely [Fig. 2(h)], and reemerges abruptly with an enhanced gap magnitude $\Delta$ (thus high- $T_{\mathrm{c}}$ superconductivity) in heavily $\mathrm{K}$-doped $\mathrm{FeSe} / \mathrm{SiC}$ films [Figs. 2(i) and 2(j)]. To characterize this tendency more visibly, more $d I / d V$ spectra have been measured in various $\mathrm{K}$-doped FeSe films and normalized, with one of them illustrated in Fig. 3(a). Assuming that a K adatom doses one electron into the low-lying FeSe films, we can summarize the superconductivity-induced spectral weight loss $\delta$ [redshaded region in Fig. 3(a)] as a function of electron doping level $x$ (electrons per Fe, namely, half of the $\mathrm{K}$ dosage) in Fig. 3(b). Here a large $\delta$ means the larger spectral weight loss due to the superconducting gap opening, and thus characterizes reasonably the superconductivity or $T_{\mathrm{c}}$. An unexpected phase diagram with two disconnected superconducting phases and a generally wide nonsuperconducting valley in between are visible, in clear contrast to the 


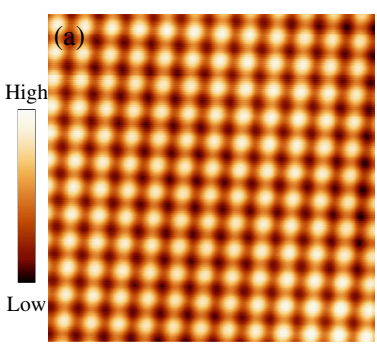

(f)

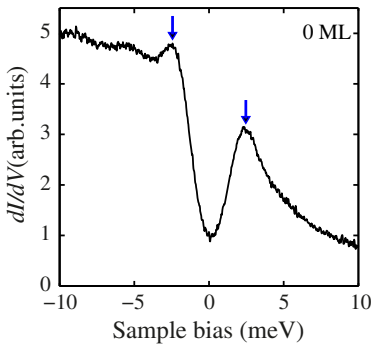

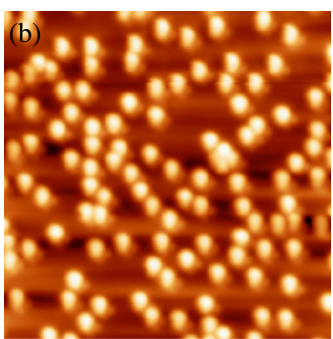

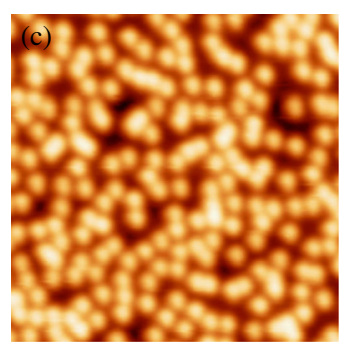

(h)

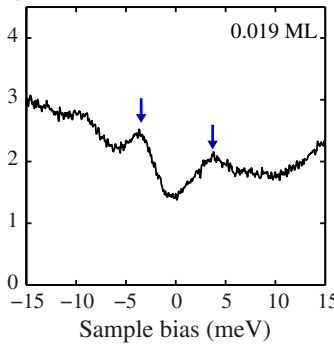

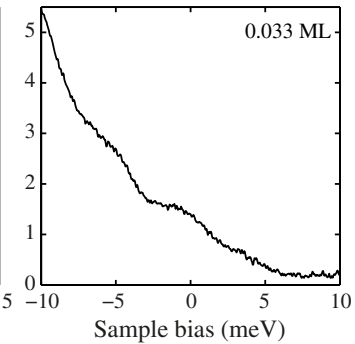

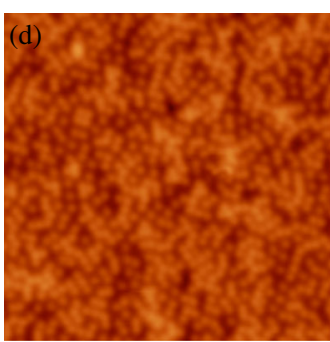

(i)

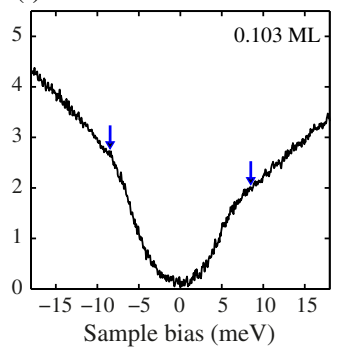

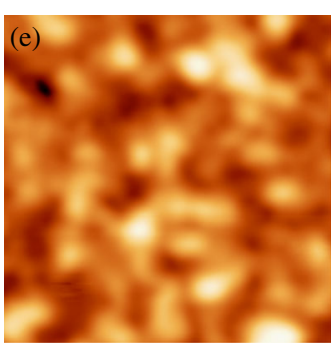

(j)

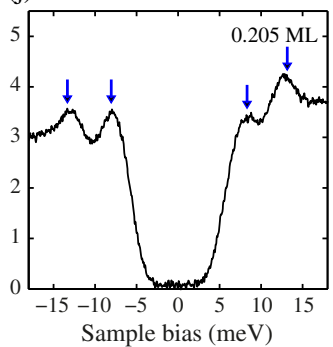

FIG. 2. (a)-(e) Topographies [(a) $5 \mathrm{~nm} \times 5 \mathrm{~nm}$. (b)-(e) $30 \mathrm{~nm} \times 30 \mathrm{~nm}]$ and (f)-(j) $d I / d V$ spectra of multilayer FeSe/SiC(0001) films with varying doses of $\mathrm{K}$, as indicated. Blue arrows denote the superconducting gap edges or coherence peaks. The absence of an apparent $E_{F}$-symmetric gap in (h) shows the full suppression of superconductivity in FeSe by an intermediate dose of K. Tunneling conditions: (a) $V=-10 \mathrm{mV}, I=100 \mathrm{pA}$; (b) $V=4.0 \mathrm{~V}, I=20 \mathrm{pA}$; (c) $V=4.0 \mathrm{~V}, I=10 \mathrm{pA}$; (d) $V=1.0 \mathrm{~V}, I=10 \mathrm{pA}$; (e) $V=3.0 \mathrm{~V}, I=20 \mathrm{pA}$; (f) $V=10 \mathrm{mV}, I=100 \mathrm{pA}$; (g) $-(\mathrm{j}) V=20 \mathrm{mV}, I=100 \mathrm{pA}$.

single-dome phase diagram reported recently [25]. This constitutes the major finding in this study.

A double-dome superconducting phase diagram has previously been identified in alkali metals and ammoniated metal-intercalated FeSe superconductors modulated by external pressure [41,42], whose mechanism so far escapes a reasonable explanation. Notably, however, the doubledome superconducting phase diagram established here differs markedly from the previous ones in terms of the external control parameter (electron doping versus pressure). Moreover, the two previous studies began from the already heavily electron-doped high- $T_{\mathrm{c}}$ phase, contrasting the present study where we start from an undoped parent
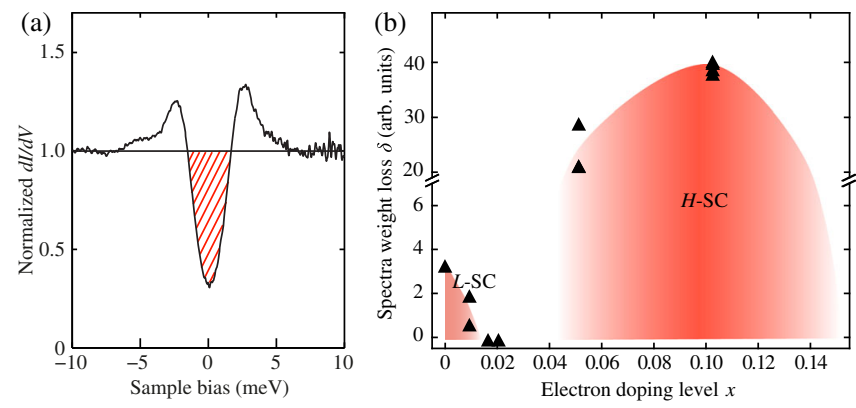

FIG. 3. (a) $d I / d V$ spectrum normalized by dividing the raw $d I / d V$ spectrum in Fig. 2(f) by its background, which was extracted from a cubic fit to the conductance for $|V|>6 \mathrm{mV}$. The red-shaded region characterizes the superconductivity-induced spectral weight loss $\delta$ near $E_{F}$. (b) Electron doping level $x$ dependence of $\delta$ (black triangles) or superconductivity, confirming two disconnected superconducting domes ( $L$-SC and $H$-SC phases) in the electron-doped FeSe phase diagram.
FeSe with a low $T_{\mathrm{c}}$ of $<10 \mathrm{~K}$. Therefore, the observation of two well-disconnected superconducting domes here, tuned by the electron doping level $x$, is intriguing and constitutes a novel experimental basis for unraveling the secret of Cooper paring in FeSe-derived superconductors.

Now the identification of two disconnected superconducting domes raises the most important concern as to whether the Cooper coupling interaction is of the same mechanism in the two disconnected superconducting domes. To bring insight into this question as well as the pairing nature of heavily electron-doped $\mathrm{FeSe}$ high- $T_{\mathrm{c}}$ superconductors, we have explored the spatial and film thickness dependence of superconductivity in heavily electron-doped FeSe films. Figure 4(a) typifies a series of $d I / d V$ spectra at various sites of the $\mathrm{K}$-doped $\mathrm{FeSe} / \mathrm{SiC}$ films, which all exhibit the two-gap feature and prove spatially homogeneous. This implies that the electrons injected by surface $\mathrm{K}$ are mostly itinerant other than localized in the $a b$ plane. In addition, the two-gap structure and gap magnitude $\Delta$ rely little on film thickness [Fig. 4(b)], unless the film is thinned down to the two-dimensional limit, namely, single-layer FeSe with a smaller single dominant superconducting gap of about $6.6 \mathrm{meV}$ [Fig. 4(c)]. This exception may be caused by the enhanced thermal or quantum fluctuations in free-standing single-layer $\mathrm{FeSe} / \mathrm{SiC}$ films, which weaken the superconductivity there [43]. Our finding that the superconducting gap shows little dependence on film thickness [Fig. 4(b)] suggests an exclusive high- $T_{\mathrm{c}}$ superconductivity at the topmost FeSe layer, consistent with recent ARPES measurements [25,40]. The gap magnitude of $\sim 14 \mathrm{meV}$ observed appears larger than most values reported in K-doped multilayer 

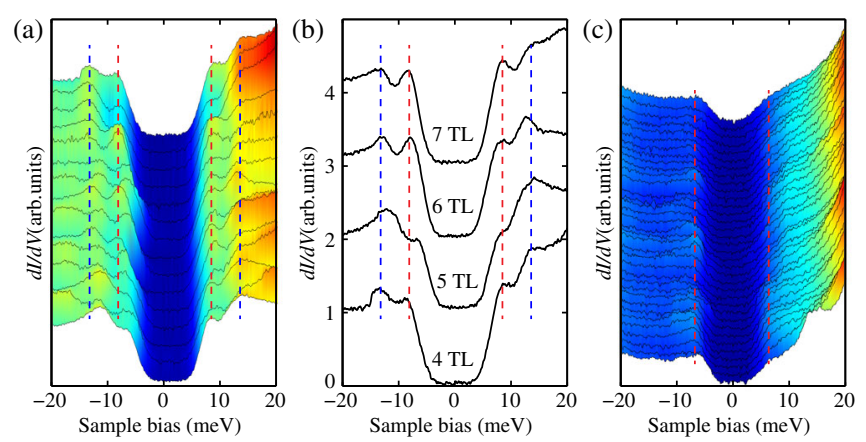

FIG. 4. (a) Spatial and (b) film thickness dependence of $d I / d V$ spectra acquired on heavily electron-doped multilayer FeSe films $(x \sim 0.103)$. Red and blue dashes show the approximate energy positions of the two energy-scale superconducting gaps, respectively. The film thickness of FeSe is indicated in a unit of triple layer (TL). (c) A series of $d I / d V$ spectra acquired along a 10-nm trajectory on heavily electron-doped single layer $\mathrm{FeSe} / \mathrm{SiC}$ films $(x \sim 0.103)$. Red dashes indicate the energy positions of the superconducting gap. Setpoint: $V=20 \mathrm{mV}, I=100 \mathrm{pA}$.

FeSe $/ \mathrm{SrTiO}_{3}$ films [24-26], but is still smaller than $\Delta \sim$ $20 \mathrm{meV}$ in single-layer $\mathrm{FeSe} / \mathrm{SrTiO}_{3}$ films [1]. Considering the smaller $\Delta \sim 6.6 \mathrm{meV}$ in K-doped single-layer FeSe/SiC films [Fig. 4(c)], our experiment corroborates that the $\mathrm{SrTiO}_{3}$ substrate plays a role that is more than electron doping to boost the high- $T_{\mathrm{c}}$ superconductivity in, or only in, single-layer $\mathrm{FeSe} / \mathrm{SrTiO}_{3}$ films, e.g., via interfacial phononenhanced pairing strength $[1,14,16,18,22]$.

Our finding of a clear separation between the two superconducting phases is seemingly not in favor of a quantum criticality picture as the key mechanism of double-dome superconductivity in FeSe [41]. In fact, the rather robust superconductivity [Fig. 4(a)] against severe disorder at the heavily K-doped FeSe surface [see Fig. 2(e)], in combination with the consistent revelations of plain- $s$-wave electron pairing in heavily electron-doped FeSe-derived superconductors [23,44], contradicts a most unconventional signchanging pairing symmetry. Instead, the findings comply with a conventional phonon-based Cooper pairing mechanism in this category of superconductors, including singlelayer $\mathrm{FeSe} / \mathrm{SrTiO}_{3}$ films [45]. This is more evidenced by the vanishing spin fluctuation in $\mathrm{Li}_{x}\left(\mathrm{C}_{2} \mathrm{H}_{8} \mathrm{~N}_{2}\right)_{y} \mathrm{Fe}_{2-z} \mathrm{Se}_{2}$ [46] and the recent tunneling measurement of the $\mathrm{FeSe} \mathrm{Eg}(\mathrm{Se})$ phonon mode $(\sim 11 \mathrm{meV})$ in K-doped multilayer FeSe/ $\mathrm{SrTiO}_{3}$ films [26], although further research efforts are needed to fully pin down this issue. Provided that the spin fluctuation picture works in parent $\mathrm{FeSe}$, two distinct paring mechanisms thus appear to operate in electron-doped FeSe films, and the heavily electron-doped ones including singlelayer $\mathrm{FeSe} / \mathrm{SrTiO}_{3}$ films are a novel class of superconductors with completely different electron pairing mechanism from $\mathrm{Fe} \mathrm{SCs}$, reflected more cogently by the contrasting properties between them. These, for example, consist of the superconducting gap function $[1,23,33,44]$, magnetic vortex core structure $[23,33]$, and nematic $[23,33,44,47,48]$ and spin fluctuations $[46,49]$. By contrast, even though the two disconnected superconducting phases are of the identical Cooper pairing mechanism, the absence of spin and nematic fluctuations in the higher- $T_{\mathrm{c}}$ superconducting phase ( $H$-SC phase) conversely supports that neither spin nor nematic fluctuations are the essential ingredient giving rise to high- $T_{\mathrm{c}}$ superconductivity $[23,44,46,48,50]$.

Finally, we comment on why the multilayer FeSe films grown on a $\mathrm{SrTiO}_{3}$ substrate exhibit no superconductivity before $\mathrm{K}$ doping, a long-standing mystery confusing the FeSe superconductivity community [1]. As seen from Fig. 3(b), the superconductivity is hypersensitive to electron doping in the lower- $T_{\mathrm{c}}$ superconducting phase ( $L$-SC phase), and an injection of only $\sim 0.015$ electrons $/ \mathrm{Fe}$ into FeSe can completely kill its superconductivity. Thus, a small amount of electron doping from the $\mathrm{SrTiO}_{3}$ substrate to multilayer FeSe films will push them to the nonsupercondcuting valley between the $L$-SC and $H$-SC phases [Fig. 3(b)]. In support of this standpoint, we have conducted a comparative study, and have found that the multilayer $\mathrm{FeSe} / \mathrm{SrTiO}_{3}$ films need a smaller $\mathrm{K}$ dose to gain high- $T_{\mathrm{c}}$ superconductivity than $\mathrm{FeSe} / \mathrm{SiC}$ films [26]. This indicates that the nonsuperconducting multilayer $\mathrm{FeSe} / \mathrm{SrTiO}_{3}$ films have already been electron doped as compared to parent FeSe.

Our detailed real-space STM or STS scrutiny of K-doped $\mathrm{FeSe} / \mathrm{SiC}$ films has demonstrated a high- $T_{\mathrm{c}}$ superconductivity at the topmost FeSe layer, and how the superconductivity evolves from the low- $T_{\mathrm{c}}$ phase in parent FeSe to the high- $T_{\mathrm{c}}$ phase in heavily electron-doped FeSe superconductors. The emergence of two disconnected superconducting domes is intriguing and will certainly stir up a number of further experimental and theoretical studies. Our work places a severe constraint on the theoretical model for understanding superconductivity in FeSe-related superconductors.

This work was financially supported by the National Science Foundation and Ministry of Science and Technology of China. C.-L. S. acknowledges support from the Tsinghua University Initiative Scientific Research Program.

*xucunma@mail.tsinghua.edu.cn †qkxue@mail.tsinghua.edu.cn

[1] Q. Y. Wang et al., Chin. Phys. Lett. 29, 037402 (2012).

[2] W. H. Zhang et al., Chin. Phys. Lett. 31, 017401 (2014).

[3] D. F. Liu et al., Nat. Commun. 3, 931 (2012).

[4] S. L. He et al., Nat. Mater. 12, 605 (2013).

[5] S. Y. Tan, Y. Zhang, M. Xia, Z. R. Ye, F. Chen, X. Xie, R. Peng, D. F. Xu, Q. Fan, H. C. Xu, J. Jiang, T. Zhang, X. C. Lai, T. Xiang, J.P. Hu, N. P. Xie, and D. L. Feng, Nat. Mater. 12, 634 (2013).

[6] R. Peng, X. P. Shen, X. Xie, H. C. Xu, S. Y. Tan, M. Xia, T. Zhang, H. Y. Cao, X. G. Gong, J. P. Hu, B. P. Xie, and D. L. Feng, Phys. Rev. Lett. 112, 107001 (2014). 
[7] Z. C. Zhang, Y. H. Wang, Q. Song, C. Liu, R. Peng, K. A. Moler, D. L. Feng, and Y. Y. Wang, Science bulletin 60, 1301 (2015).

[8] J. F. Ge, Z. L. Liu, C. H. Liu, C. L. Gao, D. Qian, Q. K. Xue, Y. Liu, and J. F. Jia, Nat. Mater. 14, 285 (2014).

[9] K. Liu, Z. Y. Lu, and T. Xiang, Phys. Rev. B 85, 235123 (2012).

[10] Y. Y. Xiang, F. Wang, D. Wang, Q. H. Wang, and D. H. Lee, Phys. Rev. B 86, 134508 (2012).

[11] J. Bang, Z. Li, Y. Y. Sun, A. Samanta, Y. Y. Zhang, W. H. Zhang, L. Wang, X. Chen, X. C. Ma, Q. K. Xue, and S. B. Zhang, Phys. Rev. B 87, 220503 (2013).

[12] S. Coh, M. L. Cohen, and S. G. Louie, New J. Phys. 17, 073027 (2015).

[13] W. H. Zhang, Z. Li, F. S. Li, H. M. Zhang, J. P. Peng, C. J. Tang, Q. Y. Wang, K. He, X. Chen, L. Wang, X. C. Ma, and Q. K. Xue, Phys. Rev. B 89, 060506 (2014).

[14] J. J. Lee, F. T. Schmitt, R. G. Moore, S. Johnston, Y. T. Cui, W. Li, M. Yi, Z. K. Liu, M. Hashimoto, Y. Zhang, D. H. Lu, T. P. Devereaux, D. H. Lee, and Z.X. Shen, Nature (London) 515, 245 (2014).

[15] L. Z. Deng, B. Lv, Z. Wu, Y. Y. Xue, W. H. Zhang, F. S. Li, L. L. Wang, X. C. Ma, Q. K. Xue, and C. W. Chu, Phys. Rev. B 90, 214513 (2014).

[16] Y. T. Cui, R. G. Moore, A. M. Zhang, Y. Tian, J. J. Lee, F. T. Schmitt, W. H. Zhang, W. Li, M. Yi, Z. K. Liu, M. Hashimoto, Y. Zhang, D. H. Lu, T. P. Devereaux, L. L. Wang, X. C. Ma, Q. M. Zhang, Q. K. Xue, D. H. Lee, and Z. X. Shen, Phys. Rev. Lett. 114, 037002 (2015).

[17] D. Huang, C. L. Song, T. A. Webb, S. Fang, C. Z. Chang, J. S. Moodera, E. Kaxiras, and J. E. Hoffman, Phys. Rev. Lett. 115, 017002 (2015).

[18] L. Rademaker, Y. Wang, T. Berlijn, and S. Johnston, New J. Phys. 18, 022001 (2016).

[19] I. I. Mazin, D. J. Singh, M. D. Johannes, and M. H. Du, Phys. Rev. Lett. 101, 057003 (2008).

[20] K. Kuroki, S. Onari, R. Arita, H. Usui, Y. Tanaka, H. Kontani, and H. Aoki, Phys. Rev. Lett. 101, 087004 (2008).

[21] P. J. Hirschfeld, M. M. Korshunov, and I. I. Mazin, Rep. Prog. Phys. 74, 124508 (2011).

[22] D. H. Lee, Chin. Phys. Lett. 24, 117405 (2015).

[23] Q. Fan, W. H. Zhang, X. Liu, Y. J. Yan, M. Q. Ren, R. Peng, H. C. Xu, B. P. Xie, J. P. Hu, T. Zhang, and D. L. Feng, Nat. Phys. 11, 946 (2015).

[24] Y. Miyata, K. Nakayama, K. Sugawara, T. Sato, and T. Takahashi, Nat. Mater. 14, 775 (2015).

[25] C. H. P. Wen, H. C. Xu, C. Chen, Z. C. Huang, Y. J. Pu, Q. Song, B. P. Xie, M. Abdel-Hafiez, D. A. Chareev, A. N. Vasiliev, R. Peng, and D. L. Feng, arXiv:1508.05848.

[26] C. J. Tang, C. Liu, G. Y. Zhou, F. S. Li, D. Zhang, Z. Li, C. L. Song, S. H. Ji, K. He, X. Chen, L. L. Wang, X. C. Ma, and Q. K. Xue, Phys. Rev. B 93, 020507 (2016).

[27] B. Lei, J. H. Cui, Z. J. Xiang, C. Shang, N. Z. Wang, G. J. Ye, X.G. Luo, T. Wu, Z. Sun, and X. H. Chen, arXiv:1509.00620.

[28] K. Hanzawa, H. Sato, H. Hiramatsu, T. Kamiya, and H. Hosono, arXiv:1508.07689.

[29] J. G. Guo, S. F. Jin, G. Wang, S. C. Wang, K. X.Zhu, T. T.Zhou, M. He, and X. L. Chen, Phys. Rev. B 82, 180520 (2010).
[30] M. Burrard-Lucas, D. G. Free, S. J. Sedlmaier, J. D. Wright, S. J. Cassidy, Y. Hara, A. J. Corkett, T. Lancaster, P. J. Baker, S. J. Blundell, and S. J. Clarke, Nat. Mater. 12, 15 (2013).

[31] X. F. Lu, N. Z. Wang, H. Wu, Y. P. Wu, D. Zhao, X. Z. Zeng, X. G. Luo, T. Wu, W. Bao, G. H. Zhang, and X. H. Chen, Nat. Mater. 14, 325 (2015).

[32] Z. Y. Du, X. Yang, H. Lin, D. L. Fang, G. Du, J. Xing, H. Yang, X. Y. Zhu, and H. H. Wen, Nat. Commun. 7, 10565 (2016).

[33] C. L. Song, Y. L. Wang, P. Cheng, Y. P. Jiang, W. Li, T. Zhang, Z. Li, K. He, L. Wang, J. F. Jia, H.-H. Hung, C. J. Wu, X. C. Ma, X. Chen, and Q. K. Xue, Science 332, 1410 (2011).

[34] C. L. Song, Y. L. Wang, Y. P. Jiang, Z. Li, L. Wang, K. He, X. Chen, X. C. Ma, and Q. K. Xue, Phys. Rev. B 84, 020503 (2011).

[35] C. L. Song, Y. L. Wang, Y. P. Jiang, Z. Li, L. Wang, K. He, X. Chen, J. E. Hoffman, X. C. Ma, and Q. K. Xue, Phys. Rev. Lett. 112, 057002 (2014).

[36] Z. Li, J. P. Peng, H. M. Zhang, W. H. Zhang, H. Ding, P. Deng, K. Chang, C. L. Song, S. H. Ji, L. Wang, K. He, X. Chen, Q. K. Xue, and X. C. Ma, J. Phys. Condens. Matter 26, 265002 (2014).

[37] C. L. Song, B. Sun, Y. L. Wang, Y. P. Jiang, L. Wang, K. He, X. Chen, P. Zhang, X. C. Ma, and Q. K. Xue, Phys. Rev. Lett. 108, 156803 (2012).

[38] C. J. Tang, D. Zhang, Y. Y. Zang, C. Liu, G. Zhou, Z. Li, C. Zheng, X. P. Hu, C. L. Song, S. H. Ji, K. He, X. Chen, L. Wang, X. C. Ma, and Q. K. Xue, Phys. Rev. B 92, 180507 (2015).

[39] Y. Zhang, J. J. Lee, R. G. Moore, W. Li, M. Hashimoto, D. H. Lu, T. P. Devereaux, D. H. Lee, and Z.X. Shen, arXiv:1512.02526.

[40] Z. R. Ye, C. F. Zhang, H. L. Ning, W. Li, L. Chen, T. Jia, M. Hashimoto, D. H. Lu, Z.X. Shen, and Y. Zhang, arXiv:1512.02526.

[41] L. L. Sun, X. J. Chen, J. Guo, P. Gao, Q. Z. Huang, H. D. Wang, M. H. Fang, X. L. Chen, G. Chen, Q. Wu, C. Zhang, D. C. Gu, X. L. Dong, L. Wang, K. Yang, A. G. Li, X. Dai, H.-K. Mao, and Z. X. Zhao, Nature (London) 483, 67 (2012).

[42] M. Izumi, L. Zheng, Y. Sakai, H. Goto, M. Sakata, Y. Nakamoto, H. L. Nguyen, T. Kagayama, K. Shimizu, S. Araki et al., Sci. Rep. 5, 9477 (2015).

[43] M. Tinkham, Introduction to Superconductivity (McGrawHill, New York, 1996).

[44] Y. J. Yan, W. H. Zhang, M. Q. Ren, X. Liu, X. F. Lu, N. Z. Wang, X. H. Niu, Q. Fan, J. Miao, R. Tao, B. P. Xie, X. H. Chen, T. Zhang, and D. L. Feng, arXiv:1507.02577.

[45] A. V. Balatsky, I. Vekhter, and J. X. Zhu, Rev. Mod. Phys. 78, 373 (2006).

[46] M. M. Hrovat, P. Jeglic, M. Klanjsek, T. Hatakeda, T. Noji, Y. Tanabe, T. Urata, K. K. Huynh, Y. Koike, K. Tanigaki, and D. Arcon, Phys. Rev. B 92, 094513 (2015).

[47] K. Nakayama, Y. Miyata, G. N. Phan, T. Sato, Y. Tanabe, T. Urata, K. Tanigaki, and T. Takahashi, Phys. Rev. Lett. 113, 237001 (2014).

[48] D. Huang, T. A. Webb, S. Fang, C. L. Song, C. Z. Chang, J. S. Moodera, E. Kaxiras, and J. E. Hoffman, arXiv:1509.07110.

[49] T. Imai, K. Ahilan, F. L. Ning, T. M. McQueen, and R. J. Cava, Phys. Rev. Lett. 102, 177005 (2009).

[50] J. Yang, R. Zhou, H. X. Wei, Lin Linand Yang, J. Q. Li, Z. X. Zhao, and G. Q. Zheng, Chin. Phys. Lett. 32, 107401 (2015). 